\title{
Die Interaktions(un)ordnung in der Mit-Corona-Gesellschaft
}

\author{
Michaela Pfadenhauer
}

Die Effekte der staatlichen Verordnung, Abstand voneinander zu halten, auf Sozialität und Gesellschaft werden soziologisch derzeit erst abgesteckt. Deutlich Distanz zu wahren, wenn Menschen räumlich aufeinandertreffen, fordert die von Erving Goffman (1971) als für gesellschaftliches Miteinander unabdingbare Interaktionsordnung heraus, weil die jeweils als üblich habitualisierten Verkehrsformen Grade von Vertrautheit, Verlässlichkeit und Verschiedenheit anzeigen, die für kommunikatives Handeln konstitutiv sind. Und alles Handeln, das sozial relevant ist, ist Kommunikation, d. h. wechselseitig aufeinander bezogen, Ausdruck des einen und Eindruck des anderen Akteurs zugleich, mit Auswirkungen von nicht nur unmittelbar sozialer, sondern mindestens mittelfristig gesellschaftlicher Bedeutung (Knoblauch 1994; 2017). Die Unordnung der Interaktionsordnung resultiert überdies aus der starken Suggestion, dass sich die für formelle Kontakte symptomatische Distanz in der Online-Begegnung reduziert hat, ohne dass Statusunterschiede und Machtgefälle im gegenwärtig laufenden Sozialexperiment ausgesetzt wären (vgl. Pfadenhauer 2021a).

\section{Leben in der Corona-Gesellschaft - ein Sozialexperiment?}

Wenn man will, kann man (auf die Gefahr hin, des Zynismus bezichtigt zu werden) COVID-19 als ein Geschenk an die Soziologie betrachten. Denn wir waren alle Zeug*innen eines breit angelegten Realexperiments, mit dem alle Nationalstaaten auf die ab einem gewissen Verbreitungsgrad exponentiell steigenden Infektionsraten flächendeckend mit Hygiene-Vorschriften bis hin zum totalen Lockdown reagiert haben.

Wie in einem Experiment haben wir bis heute den Untersuchungsraum kaum mehr verlassen, der allen Europäisierungsschritten zum Trotz auf einmal wieder wie selbstverständlich national abgesteckt ist. Rechtlich lässt sich das nachvollziehen, weil das aktuelle COVID-19-Maßnahmengesetz z. B. auf dem Epidemiegesetz von 1913 beruht. Politisch ist kaum nachvollziehbar, dass die Europäisierung der vergangenen Jahrzehnte sich erst beim Impfgeschehen bemerkbar gemacht hat.

Der Logik des Experiments folgt auch die Überprüfung der erlassenen Maßnahmen alle zehn bis vierzehn Tage, um deren Wirksamkeit an der 
Entwicklung der Infektionszahlen zu überprüfen. Diese Logik befördert die so genannte „exekutive Politik“, die in Krisen im Vorteil ist (Vobruba 2020, 145). Gemeint ist damit, so die Juristin und Rechtssoziologin Monika Frommel (2021), „eine ohne präzise parlamentarische Vorarbeit über Verordnungen verbindlich handelnde Exekutive. Die jeweiligen Maßnahmen sind schwer kontrollierbar, weil die gesetzlichen Grundlagen schwach und ungenau sind. Gerichten fehlen deshalb die Prüfungsmaßstäbe“. Exekutive Politik geht somit gewissermaßen im Eilschritt an der Legislative vorbei. Begleitet wird sie dabei von einem Diskurs, der Expertokratie/Technokratie befürchtet: In den 1970er Jahren eher von linksliberalen Intellektuellen befeuert, findet sich der Alarmismus, wir würden von einer Expert*innen-Elite regiert, die mit einem Regierungs-Medien-Komplex verschmolzen sei, als fester Bestandteil im populistischen Repertoire.

Politikwissenschaftler*innen wie Andreas Busch (2021) zufolge war bzw. ist diese Politik keine Expertokratie im demokratisch illegitimen Sinn. Sie erfolgt ausgesprochen evidenzbasiert, d. h. auf der Basis wissenschaftlicher Erkenntnisse, insbesondere der Epidemiologie und Virologie sowie statistischer Modellrechnungen, erst später dann auch der Medizin. Im Drei-Länder-Vergleich war das in Deutschland vorherrschende Institutionen-gestützte System der wissenschaftlichen Politikberatung relativ zu Großbritannien und den USA gesehen das erfolgreichste. Österreich ist nicht ganz mit Deutschland vergleichbar mit Institutionen ausgestattet, weshalb hier der Ruf nach einem Robert-Koch-Institut zu hören war, das in Deutschland allerdings auch immer wieder in die Kritik geraten ist. Jedoch hat die institutionell gebündelte Expertise schneller wirksam werden können als dies in Systemen der Fall war, die stattdessen auf individuelle Strategien wie Chefberater"innen und persönlich zusammengesetzte Think Tanks gesetzt hatten, sofern sich Regierungen nicht überhaupt weitgehend gegen Expertise abgeschottet hatten.

Dem Politologen Simon Hegelich zufolge ist diese evidenzbasierte Politik allerdings mit abnehmender Evidenz, dass die Maßnahmen wirken, in eine Glaubensfrage umgeschlagen. Dies zeigte sich in einem jegliche Kritik zurückweisendem Festhalten am Narrativ „Die Maßnahmen wirken!“. Die Kritikempfindlichkeit dieses Narrativs ist angesichts dessen, dass "Kritik einen ganz normalen Platz im öffentlichen Diskurs hat" (Hegelich 2021, 306), bemerkenswert. Und Kritikresistenz zeitigt Folgen: Zum einen treibt sie die Gesellschaft auseinander; zum anderen werden Ursachen für steigende Infektionszahlen nicht mehr strukturell, sondern individuell gesucht, d. h. es sind „Einzelne, die sich am gesellschaftlichen Auftrag vergehen" (Hegelich 2021, 309) - Jugendliche, die Partys feiern; Betuchte, die ihre Zweitwohnsitze aufsuchen; Familien mit Migrationshin- 
tergrund, die an hohen Feiertagen ihre Herkunftsorte aufsuchen. Besonders problematisch ist es, wenn diese individuelle Schuldzuweisung mit parteipolitischem Kalkül seitens Regierungsvertreter*innen erfolgt, weil dies sozusagen einen Keil weiter in die Gesellschaft hineintreibt. Auch hier ist es bemerkenswert, dass dies in den ersten Wochen der Pandemie nicht erfolgt ist; erst mit der Rückkehr aus dem Sommerurlaub im Verein mit steigenden Infektionszahlen waren politische Stimmen zu hören, dass das Virus von außen, eingeschleppt ${ }^{6}$ worden sei.

Im Rahmen dieses gleichsam im Zweiwochenrhythmus nachjustierten realen Sozialexperiments konnten wir die Wirkung neuer Regeln des sozialen Umgangs ebenso wie jene der Irritation von Routinen des Arbeitens teilnehmend beobachten, - gewissermaßen wie in einem Experiment auf Zeit, denn die Rückkehr der bisherigen Normalität war für uns selbstverständlich, weil wir von einem ,Ausnahmezustand' ausgegangen sind. Dies war politisch im Übrigen zu keinem Moment der Fall, in gewisser Weise kann - wovon gleich noch die Rede sein wird - sogar von einer Ermächtigung der Bürger*innen die Rede sein. Dennoch war dieser Eindruck eines Einbruchs, bei dem man nur warten muss, bis er vorübergeht, lange Zeit unabweisbar. Das lag und liegt nicht zum wenigsten daran, dass von der Kommunikation der Maßnahmen her darauf verzichtet worden ist und wird, die bereits prognostizierbare zeitliche Ausdehnung der Infektionsentwicklung und die aufgrund von Mutationen sowie ebenso wegen der nachlassenden Immunisierungswirkung von Impfungen prinzipielle Unabgeschlossenheit zu thematisieren.

Im Rahmen dieses Großexperiments ist soziologisch zu fragen, ob die neuen Umgangsformen wie der Ellenbogenschlag und Faustgruß im Verzicht auf Händeschütteln und Umarmen oder das hierzulande sehr ungewohnt geordnete Schlangestehen vor Geschäften die Interaktionsordnung, also die Ordnung, wie sich Menschen begegnen, auf Dauer ändern werden. Beobachtbar ist derzeit nach wie vor eine Irritation im Umgang miteinander, eine Inkompetenz kommunikativen Handelns, etwa was das Einschätzen von Verlässlichkeit des Gegenübers angeht, weshalb das Social Distancing bzw. Distant Socializing eine allgemeine Verunsicherung im Umgang insbesondere Unbekannter miteinander evoziert.

Wir (Soziolog*innen) fragen uns, welche Konsequenzen die mit der weitgehenden Aussetzung der meisten wirtschaftlichen, sozialen und kulturellen Aktivitäten einhergehende Umstellung auf digitale Kommunikationskanäle zeitigt, die in privaten (Interaktionen in der Großfamilie), beruflichen (Arbeit zu Hause) und öffentlichen (religiöse Zeremonien) Situationen stark genutzt wurden. Wie tragen videogestützte Interaktionen zur Aufrechterhaltung der Sozialität während der Pandemie bei? Beobacht- 
bar ist derzeit, welche Bedeutung den Institutionen zur Aufrechterhaltung des Betriebs beizumessen ist, insbesondere auch Zeitinstitutionen wie gemeinsame Kaffeepausen, wöchentliche Besprechungen, monatliche Institutsversammlungen etc. In Zeiten von Lockdown und Homeoffice sind Organisationen privatwirtschaftlicher oder öffentlicher Natur nur dadurch als existent erfahrbar (vgl. grundlegend Hitzler/vom Lehn 2019).

Dies kann in diesem realen Sozialexperiment wie unter dem Brennglas beobachtet werden. Allerdings fehlt die systematische Kontrolle der Randbedingungen wie im Labor, weshalb jeweils bestimmte Folgen anders als bei einer künstlichen, detailliert vorausgeplanten Untersuchungsanlage nicht exakt auf ihre jeweiligen Ursachen zurückgeführt werden können: Worin gründet etwa die abnehmende Bereitschaft zur Einhaltung der Hygiene-Auflagen? In Fehlern der Krisenkommunikation, wie medial diskutiert, oder aber in abnehmender Solidarität, was zumindest für die von Michael Corsten und Hanna Haag (2021) untersuchte „Generation Corona“, d. h. die erste volle Social-Web-Generation, nicht haltbar zu sein scheint? Was sind die Ursachen für die abnehmende Impfbereitschaft, die das Austrian Corona Panel Project belegt (vgl. Kittel 2020)? Daten von Johannes Weyer (2020) geben Anlass zur Vermutung, dass Corona-Skepsis und Parteizugehörigkeit korrelieren. Damit wäre das Commitment zu Maßnahmen der Regierung zumindest auch ein Ausdruck von Parteizugehörigkeit, nämlich zu einer jener Parteien, die die Regierung in der Krise stellen. Dies ist kein Problem, solange die Parteien fast wie in einer Expert*innen-Regierung die Maßnahmen gemeinsam tragen. Parteienstreit aber befördert Skepsis und spaltet die Gesellschaft, weshalb eine parlamentarische Auseinandersetzung mit dann von allen getragenen Beschlüssen das demokratische Mittel der Wahl wäre.

Besonders auffällig war bei diesen Bemühungen um flächendeckendes Commitment, dass es nicht Ärztinnen und Ärzte, sondern Repräsentant*innen der Politik waren, die uns Maßnahmen zum Schutz der Gesundheit erklärt haben, was Frommel (2020) als „Volkspädagogik“ bezeichnet hat. Nicht nur werden wir dergestalt von Politiker*innen statt von Ärzten und Ärztinnen als Patient*innen adressiert (vgl. nochmals Pfadenhauer 2021a); die Kontaktbeschränkungen und Distanzregeln, insbesondere aber der Mund-Nasen-Schutz versetzt uns in die Rolle von (zumindest potenziell) Kranken. Nun ist die Krankenrolle - rollentheoretisch betrachtet - nicht mit Hilflosigkeit und Passivität zu verwechseln, sondern impliziert den aktiven Part des Wiedererlangens oder Erhaltens von Gesundheit, den die meisten von uns bereitwillig übernommen haben. Professionssoziologisch ist diesbezüglich von einem "Arbeitskonsensus“ (Goffman 1971, 17) zwischen professionell Tätigen und Klient*innen die 
Rede (Pfadenhauer 2003, 150). Die Krankenrolle impliziert aber eben auch den Bedarf an stellvertretender Deutung durch die ärztliche Profession, die im Falle des Rat-Befolgens zum Gesundheitsstatus zurückführt.

Der Übergang von Gesundheit zu Krankheit (und zurück) kann als Ritualprozess beschrieben werden, wie er sozialanthropologisch als „rite des passage“ (van Gennep 1960/1909) konzipiert worden ist. Demnach liegt zwischen dem Ritus der Absonderung und der Wiedereingliederung in die Sozialordnung eine Schwellenphase, die mit einem Statusverlust (zum Beispiel Kind/nicht mehr Kind, gesund/nicht mehr gesund) einhergeht, ohne dass der neue Status (erwachsen bzw. wieder gesund) schon erreicht ist. Ausdrucksstarkes Symbol für diese Übergangsphase, in der sich die Mit-Corona-Gesellschaft derzeit befindet, ist die Maske, die uns im Sinne dessen, was Victor Turner (2008) als „liminal“ bzw. „liminoid“ bezeichnet, vergemeinschaftet, wobei es sich in komplex strukturierten Gesellschaften wie der unseren maximal um eine zeitlich begrenzte „Vergemeinschaftung" (Hitzler et al. 2008) handeln kann. Schon Turner legt communitas prozesshaft bzw. Gesellschaft als ein „System kommunizierender Röhren an: Wird die Gleichheit der Gemeinschaft betont, so entsteht unweigerlich ein Druck, mehr Struktur zu schaffen“ (Förster 2003, 7).

Diese Struktur wird in Österreich derzeit als „3G“ kommuniziert: Neben die Genesenen treten die Getesteten und in der letzten Zeit vermehrt auch die Geimpften. Für alle aber gilt, dass der Status vergänglich ist: am klarsten ist das (im Sinne von einsichtig ebenso wie diskutiert) - bei den Getesteten, deren Zustand nur eine Momentaufnahme ist. Die beiden anderen Gruppen sind unklar umrissen: wie lange hält die Immunisierung durch Erkrankung, wie lange die durch die Impfung - und das auch noch unterschiedlich je nach Impfstoff?

\section{In der, Schule des Vergleichens}

Bleibt man in der Logik des Experiments, kann man es in methodischer Hinsicht enttäuschend finden, dass sich die exekutive Politik der Länder immer mehr einander angenähert hat. Denn für ein methodologisch einwandfreies Ergebnis wären Kontrollgruppen das Mittel der Wahl: also mindestens ein Staat, der vollständig auf Maßnahmen verzichtet, während anderswo deutlich abweichende Mittel zur Pandemiebekämpfung eingesetzt werden als im Mainstream der Länder (für Europa war Schweden so ein Fall, wobei die mediale Berichterstattung weit mehr das Annähern als die Abweichung verbreitet hat).

Ein solcher methodologisch-methodischer Blick auf Corona mutet zynisch an, fördert aber zutage, dass Staaten nicht wirklich vergleichbar sind, 
weil sich ihre Gesundheitssysteme ebenso wie ihre Kulturen im Hinblick auf Tradition und Geselligkeit deutlich unterscheiden. Ivan Krastev (2020, 63) beschreibt diese Strategie-Anpassung als auf Ungewissheit reagierende „Nachahmungspolitik“. Als Nährboden für diese unter dem Gesichtspunkt der Nichtvergleichbarkeit unverständliche und auch problematische Nachahmungspolitik sieht er die Echtzeit-Beobachtung, unter der sich Regierungen wähnten: Die täglich bereits im Ländervergleich gemeldeten Zahlen vermitteln uns Bürger*innen den Eindruck, die Wirkung von Maßnahmen vergleichend beurteilen zu können.

Das Paradoxon der Corona-Krise liegt darin, dass sie nicht nur die Staaten durch außerordentliche Vollmachten ermächtigt hat, sondern auch jedem einzelnen Bürger eine gewisse Ermächtigung verleiht: durch die Möglichkeit, zu urteilen, ob seine Regierung sich besser oder schlechter schlägt als andere. (Krastev 2020, 64)

Dementsprechend ist der Historikerin Angelika Epple (2020, 25) recht zu geben, dass „wir seit Corona in der Schule des Vergleichens [leben]. [...] Wir sind Augenzeug*innen, wie mithilfe von Vergleichen Orientierung gesucht, gefunden und begründet wird. Methodische Finessen des statistischen Vergleichens werden diskutiert, die Aussagekraft der Zahlen angezweifelt, um anschließend weiter zu vergleichen“. Problematisch ist daran einerseits das, was Epple (2020,32) „Objektivismus“ nennt, das heißt die Überbewertung der Objektivität der Vergleiche, die sie derzeit beim politischen Vergleichen am Werke sieht; andererseits „die Überbetonung des Konstruktivismus“. Hier wird der Umstand, dass Vergleiche immer einen Konstruktionsaspekt implizieren, mit Beliebigkeit verwechselt, was „die Gefahr der Verschwörungstheorien und fake news [birgt]“" (Epple 2020,32).

Diese unser aller Vergleichsperspektive hat einen Globalisierungseffekt: Üblicherweise verstehen wir unter Globalisierung ,grenzüberschreitende Verbindungen“ (Heintz 2016, 318). Bettina Heintz bezeichnet neben „strukturellen Vernetzungen“ auch Vergleiche als „Globalisierungsmechanismus“. Dabei handelt es sich auch um Verknüpfungen, die auf einer kulturellen Ebene angesiedelt sind, insofern die ,verglichenen Einheiten über ein Vergleichskriterium in einen Sinnzusammenhang gebracht werden“. Ihr Beispiel sind internationale Hochschulrankings. Aus der Forschung wissen wir, dass Hochschulsysteme nicht wirklich vergleichbar sind, weil die Bedingungen in den einzelnen Ländern sehr unterschiedlich sind - zuvorderst wegen den Studiengebühren, die Universitäten sehr unterschiedlich finanzstark machen. Universitäten nehmen sich seither aber 
als Wettbewerber wahr, wobei die früher begrenzte, lokale Konkurrenz generalisiert wird.

Da in ihnen häufig das Vergleichsmoment gebündelt wird, kommt globalen Zahlen, aktuell dem Inzidenzwert, eine besondere Wirkmacht zu. Aber Zahlen sind nur die Form, wie der Vergleich kommuniziert wird. Über diese „Kommunikationsform“ (Heintz 2016, S. 317) hinaus ist es der Vergleichszusammenhang selbst, der Wirkung entfaltet: nämlich Anpassung ohne weitere Steuerungsmaßnahmen im Falle von Universitäten bzw. Nachahmungspolitik im Falle von Regierungen.

Das Real-Experiment tangiert mit Contact Tracing einerseits, mit Ausgangssperren andererseits den zumindest von staatlichem Eingriff her weitgehend als sicher gehaltenen Schutz des Privaten: Zum einen wird das, was im privaten Nahraum passiert, selbst dann, wenn den Ordnungskräften ein Zugriff verboten ist, zum Gegenstand öffentlich-staatlichen Interesses; zum anderen ist das Patient*innen-Geheimnis berührt, wenn die Infektion einer Person meldepflichtig wird und im Zuge einer ,Kontaktverfolgung' nicht nur Bewegungen in Raum und Zeit, sondern das Sozialverhalten zur Disposition steht (wer macht was wann wo - mit unangenehmen Formen des Vernaderns), wie Fälle derart angeprangerter Super-Spreader deutlich machen.

Die Corona-App ist die in der Logik einer Gesellschaft, die Steffen Mau (2017) der Logik des Vergleichens entsprechend als "metrisches Wir“ beschrieben hat, die folgerichtige Antwort. Von dieser Seite her schien uns unter dem Gesichtspunkt des Datenschutzes der Schutz der Privatsphäre geboten, wobei fraglich ist, ob mehr Gefährdung vom demokratischen Staat oder von den privatwirtschaftlichen Interessen kommerzieller Konzerne ausgeht, gepaart mit unser aller freigiebigen Bereitschaft zur Preisgabe der Daten. Die Angst davor, zum ,gläsernen Bürger` bzw. zur, gläsernen Bürgerin' zu werden, hat dazu geführt, dass sich im Zuge zäher Verhandlungen, die in der Zeit vom 18. Juni 2020 als „Digitalexperiment“ beschrieben werden, eine Koalition aus „Weltkonzernen mit deutschen Hackern, IT-Forschern und Datenschützern gegen die zentrale Datenspeicherung“ gebildet hat, in deren Folge sich in Deutschland (ebenso wie in Österreich und vielen anderen Ländern) eine dezentrale und datenschutzfreundliche Lösung durchgesetzt hat.

Warum aber hat sich deren Nutzung trotz gesundheitspolitischer Werbebotschaften nicht flächendeckend verbreitet? Gerade im Hinblick auf Digitalisierung definiert die EU Ansprüche an den Datenschutz, der für Konzerne weltweit Standards setzt, was Ann Bradford (2020) als „BrüsselEffekt“ bezeichnet hat. Die Zurückhaltung gegenüber der Nutzung der Corona-App in vielen europäischen Ländern zeigt, dass dieser Effekt mit 
der von Armin Nassehi (2019) als „Unbehagen an der digitalen Kultur“ bezeichneten Kulturkritik Hand in Hand geht, die sich - anders als von Nassehi (2020) gemutmaßt - trotz aller Videokonferenz-Euphorie auch unter Bedingungen der Pandemie nicht in ihr Gegenteil verkehrt.

\section{Die Corona-Pandemie - anders als frühere Pandemien?}

In jeder anderen Hinsicht war Digitalisierung die uneingeschränkt akzeptierte Lösung nicht nur beim Homeschooling und -teaching sowie im Homeoffice, sondern auch bei der täglichen gegenseitigen Begleitung in Chats und Social Media Groups aller Art. Ähnlich wie millionenfach verschickte Kettenbriefe mit Aufrufen zu Solidarität, die auch nach $\mathrm{Na}$ turkatastrophen üblich sind, sind Solidaritätsbekundungen eine - nun in digitalem Gewand - altbekannte Antwort auf die als ein gänzlich neues Phänomen wahrgenommene Pandemie. Insbesondere beim ersten Shutdown im März/April 2020 war der Eindruck des Noch-nie-Dagewesenen atemberaubend.

Wie uns der Blick zurück auf die Spanische Grippe vor hundert Jahren und auf die vom 14. bis zum 18. Jahrhundert in Europa wütende Pest lehrt, sind weder Pandemien noch die aktuell angewendeten Strategien zu deren Bewältigung historisch neu oder „dem autoritären China abgeschaut. Ausgangsverbote, Quarantäne, das Herunterfahren wirtschaftlicher Aktivitäten, Versammlungsverbote, selbst das Abschotten ganzer Städte und Regionen, wie in Norditalien, sind Erbe der europäischen Tradition im Umgang mit Epidemien“ (Mauelshagen 2020, 38).

Auch nicht neu ist, dass der aktuelle ebenso wie die Krankheitserreger früherer Zeiten grundsätzlich erstmal keine Rang- und Statusunterschiede kennt. Dies hat die Mär vom Virus als Gleichmacher beflügelt, bevor erkennbar wurde, dass zwar jede und jeder angesteckt werden kann, es aber einen gravierenden Unterschied macht, ob das Virus ein wohlbehütet isolierbares Individuum möglicherweise gar öffentlichen Interesses befällt oder ob es sich bei fließbandartigen Arbeitsbedingungen in engen Fabrikhallen unter der gesamten Belegschaft verbreitet, die zudem beengte Wohnverhältnisse teilen, unter denen die Quarantäne für jede*n Einzelne*n existenzgefährdend sein kann.

Jedoch auch ohne absoluten Neuigkeitswert ist die Einordnung der Corona-Pandemie als Krise nicht zu hoch gegriffen, weil Prozesse zu gewärtigen sind, die „die ,Grammatik' sozialer Prozesse verletzen“ (Offe 1973, 199). ,Krise‘ als alltäglich und medial inflationär gebrauchten Begriff reserviert Joris Steg $(2020$, 432) für „Phasen, in denen die Struktur, die Funktionsfähigkeit oder der Bestand [...] eines sozialen Zusammenhangs 
gefährdet sind“. Zum einen dürften die Strategien der Pandemieeindämmung eine erhebliche Verschärfung sozialer Ungleichheit nach sich ziehen, die manche Beobachter*innen auf eine endgültige Spaltung der Gesellschaft zulaufen sehen. Zum anderen sind Verletzungen der Prozesse der sozialen Grammatik auf der Ebene kommunikativer Prozesse zu beobachten.

Über seine unmittelbare Funktion hinaus ist der Mund-Nasen-Schutz nicht nur eine permanente Gedächtnisstütze, in diesem Sinne also ganz unmittelbar eine Storage Unit (Turner 2008 [Erstausgabe 1968], 1f.), sondern Ausdruck einer Statusdegradierung zwischen gesund und krank, der uns mit gravierenden Konsequenzen für die bisherige Interaktionsordnung maskiert und in unseren bisher gängigen Ausdrucksmöglichkeiten beschränkt (vgl. auch Löw/Knoblauch 2020). Die Elemente einer neuen Interaktions- und Sozialordnung sind noch nicht ausgemacht.

Die Kehrseite der exekutiven Politik ist das Warten auf amtliche Verordnungen, die kaum nachkommen und nicht alle Fälle regeln können, ganz abgesehen davon, dass diese Verrechtlichung nicht erstrebenswert ist. Wie aber regeln wir dann - mit Höflichkeit, gesundem Menschenverstand, offenen Absprachen - unser Miteinander?

\section{Gewissheit war früher}

Die gegenwärtige Krise, die sich allein in ihrer akuten Phase noch weit in 2021 hineinzieht, ist einer jener

Momente, in denen sich unsere Gewissheiten auflösen und sich unsere kollektive Vorstellung von dem, was möglich ist, dramatisch ändert. Die Menschen beginnen, die Gegenwart zu ignorieren und stattdessen über die Zukunft nachzudenken - die Zukunft, die sie sich erhoffen, oder die Zukunft, die sie fürchten. (Krastev 2020, 22)

Dies entbehrt nicht einer gewissen Ironie. Denn die Pandemie - hier bezieht sich Krastev $(2020,22)$ auf Die Pest von Albert Camus - „löschte ,die Erfahrung des persönlichen Lebens' aus, indem sie einem jeden die eigene Verletzlichkeit und Machtlosigkeit, die Zukunft zu planen, vor Augen führte“.

Der Historiker Benjamin Scheller weist darauf hin, dass sich frühere Pandemien wie der Schwarze Tod nur begrenzt als Vorbilder eignen. Am häufigsten diente - auch seitens Epidemiolog*innen und Virolog*innen - die Spanische Grippe von 1918 bis 1919 als Vergleichsmaßstab. Bettina Heintz beschreibt in ihrer Soziologie des Vergleichens Analogien als „Ordnungstechniken, die zwischen dem Entferntesten Ähnlichkeiten kon- 
statieren: jedes Element findet in anderen Elementen seine Entsprechung. Unterschiede werden abgeschwächt und in ein System von Homologien eingeordnet". Analogiebildungen sind heute deshalb der Scheinargumentation verdächtig und unterliegen der wissenschaftlichen Beweispflicht, mit der sie sich selten als haltbar erweisen; dennoch haben sie während der Corona-Pandemie eine nicht unerhebliche Rolle gespielt: So soll es der Bezug auf die historische Analogie der Spanischen Grippe gewesen sein, die der Virologe Christian Drosten im März 2020 nach der Lektüre einer Studie zu dieser Grippe während einem Zusammentreffen mit Repräsentanten der Regierung gezogen hat, der die schnelle allgemeine Schulschließung in Deutschland zur Folge gehabt habe. Die Geschichte biete aber keine Präzedenzfälle, so Scheller (2021, 263), „aus ihr lasse sich vor allem lernen, eine Zukunft zu erwarten, die völlig anders sein wird als die Vergangenheit und sich deren Andersartigkeit wenigstens in groben Umrissen vorzustellen“.

Historische Analogiebildung ist eine Form „reaktiver Kontingenzbewältigung" (Scheller 2021, 259) unter Bedingungen, in denen uns mangels Erfahrungen die Möglichkeit abhandengekommen ist, Erwartungen im Hinblick auf die Zukunft auszubilden. Wenn der Begriff der Erwartung - mit Reinhart Kosellek $(1979,255)$ gesprochen ist Erwartung „vergegenwärtigte Zukunft“ - immer ein „Moment der Gewissheit zum Ausdruck“ bringt (Luhmann 1983, 362), dann zeugen die Analogien, so hinkend sie sein mögen, vor allem von Ungewissheit. Denn "Quellen von Gewissheit“, wie Angelika Poferl (2018) Helmuth Plessner zitiert, liegen nicht im Denken, in der reinen Vernunft und der Wissenschaft, sondern in körperlichsinnlichen, gegenstandsbezogenen Erfahrungen der Lebensweltlichkeit des Alltags.

Von daher werden wir nicht in einer Post-Corona-Gesellschaft, sondern in einer Mit-Corona-Gesellschaft leben. Denn nicht nur wird das Covid-19Virus in seiner Wechselbalg-Gestalt Teil unserer Wirklichkeit bleiben. Die Erfahrung unseres Umgangs damit wird Teil unseres gesellschaftlichen Wissensbestands sein und sich auch tief ins kollektive wie individuelle Bewusstsein eingeschrieben haben. Daraus wird sie gelegentlich - eher zu unpassenden Gelegenheiten - wiederauftauchen und uns einen Anflug von Unsicherheit spüren lassen, wie ein kalter Zug, der einen in der Situation so unmerklich anweht, dass man ihn kaum lokalisieren kann, aber einen steifen Hals beschert, an dem man lange laboriert. Gut vorstellbar ist, dass wir das Abhandenkommen von Zukunft, das Camus als beklemmende Erfahrung einer ,geschlossenen Gesellschaft ${ }^{\star}$ beschrieben hat, durch hohen Planungseifer kompensieren werden. Aber in vielen unserer $\mathrm{Zu}-$ kunftsentwürfe wird eine Verunsicherung mitschwingen, die daher rührt, 
dass wir in unserer nachgerade bräsigen Gewissheit erschüttert wurden. Für unsereins unvorstellbar mussten wir erfahren, dass Gewissheiten des Und-so-Weiter und Ich-kann-immer-Wieder (Schütz/Luckmann 1979, 72), derer wir vor der Pandemie so überdrüssig waren, dass wir gern aus ihnen ausgebrochen wären, nie mehr als ein Taken-for-granted, $d$. h. lebensweltliche Idealisierungen, sind (vgl. grundlegend Pfadenhauer 2021b).

In diesem Sinne werden gerade wir individualisierten Akteur*innen, die wir alle Weichenstellungen unseres Lebens ausschließlich von unseren persönlichen Entscheidungen abhängig sahen, so manches als nicht mehr fraglos garantiert ansehen: unseren vor staatlichem Eingriff geschützten Nah-Raum etwa. Wir müssen uns deshalb darauf einstellen, dass mit der Ungewissheit der Mit-Corona-Gesellschaft bei allem vielleicht überstürzten Rückfall in alte Gewohnheiten die allgemeine Verunsicherung, die viel existenzieller ist als die einer postmodernen Unübersichtlichkeit, uns deshalb für geraume Zeit begleiten wird.

\section{Literatur}

Bradford, Anu: The Brussels Effect. How the European Union Rules the World, Oxford: Oxford University Press 2020.

Busch, Andreas: Wissen allein genügt nicht. Die Nutzung von politikberatenden Institutionen während der Corona-Pandemie im Vergleich, in: Florack, Martin/ Korte, Karl-Rudolf/Schwanholz, Julia (Hg.): Coronakratie. Demokratisches Regieren in Ausnahmezeiten, Frankfurt/New York: Campus 2021, 283-293.

Corsten, Michael/Haag, Hanna: Was soll das Gerede von der „Generation Corona"?, in: Soziologische Perspektiven auf die Corona-Krise. Digitales Kolloquium am 11.02.2021, Wissenschaftszentrum Berlin für Sozialforschung, https://corona soziologie.blog.wzb.eu/podcast/michael-corsten-und-hanna-haag-was-soll-das-ger ede-von-der-generation-corona/.

Endreß, Martin/Niessen, Silke/Vobruba, Georg: Aktualität der Demokratie, Weinheim: Beltz Juventa 2020.

Epple, Angelika: Die Schule des Vergleichens und die Suche nach der Wahrheit wissenschaftlicher Fakten, in: Volkmer, Michael/Werner, Karin (Hg.): Die Corona-Gesellschaft. Analysen zur Lage und Perspektiven für die Zukunft, Bielefeld: Transkript 2020, 25-33.

Förster, Till: Victor Turners Ritualtheorie, in: Theologische Literaturzeitung 128, 7/8 (2003) 703-716.

Frommel, Monika: Corona-Politik 2020 - ein schmaler Grat zwischen kluger Einschränkung und überzogener Panik, in: Neue Kriminalpolitik (H. 2/2020) 113123.

Frommel, Monika: Die zweite Welle der Corona-Politik - ein Rückblick. Unveröffentlichtes Manuskript 2021. 
van Gennep, Arnold: The Rites of Passage, Chicago/London: University of Chicago Press 1960.

Goffman, Erving: Interaktionsrituale. Über Verhalten in direkter Kommunikation, Frankfurt am Main: Suhrkamp ${ }^{4} 1971$.

Hegelich, Simon: Evidenzbasiertes Regieren: Von klaren Zielvorgaben in der Corona-Politik zur Glaubensfrage, in: Florack, Martin/Korte, Karl-Rudolf/Schwanholz, Julia (Hg.): Coronakratie. Demokratisches Regieren in Ausnahmezeiten, Frankfurt/New York: Campus 2021, 295-310.

Heintz, Bettina: „Wir leben im Zeitalter der Vergleichung.“ Perspektiven einer Soziologie des Vergleichs, in: Zeitschrift für Soziologie 45/5 (2016) 305-323.

Hitzler, Ronald/vom Lehn, Dirk: Organisation als subjektive Erfahrung. Ein Phänomenologie-basierter Beitrag in konzeptionell ergänzender Absicht, in: Pfadenhauer, Michaela/Scheibelhofer, Elisabeth (Hg.): Interpretative Sozial- und Organisationsforschung, Weinheim: Beltz Juventa 2020, 252-269.

Hitzler, Ronald/Honer, Anne/Pfadenhauer, Michaela (Hg.): Posttraditionale Gemeinschaften. Theoretische und ethnografische Erkundungen, Wiesbaden: Springer VS 2008.

Kirschner, Heiko/Hitzler, Ronald: Die Medialisierung der Interaktionsordnung und die Ordnung mediatisierter Interaktion. Über digitale Herausforderungen des Goffmenschen, in: Lessenich, Stephan (Hg.): Geschlossene Gesellschaften. Verhandlungen des 38. Kongresses der Deutschen Gesellschaft für Soziologie in Bamberg 2016, veröffentlicht am 30.09.2017 unter https://publikationen.soziolo gie.de/index.php/kongressband_2016/article/view/527.

Kittel, Bernhard: Die Erosion der Impfbereitschaft in der österreichischen Bevölkerung. Corona-Blog 87 (2020), https://viecer.univie.ac.at/corona-blog/corona-blog -beitraege/blog87/.

Knoblauch, Hubert: Erving Goffmans Reich der Interaktion, in: Goffman, Erving (Hg.): Interaktion und Geschlecht, Frankfurt/M.: Campus 1994, 7-49.

Knoblauch, Hubert: Die kommunikative Konstruktion der Wirklichkeit, Wiesbaden: Springer VS 2017.

Kosellek, Reinhart: Vergangene Zukunft. Zur Semantik geschichtlicher Zeiten, Berlin/Frankfurt/M.: Suhrkamp 1979.

Krastev, Ivan: Ist heute schon morgen? Wie die Pandemie Europa verändert, Berlin: Ullstein 2020.

Löw, Martina/Knoblauch, Hubert: Dancing in Quarantine. The Spatial Refiguration of Society and the Interaction Orders, in: Space and Culture 23/3 (2020) 221-225.

Luhmann, Niklas: Soziale Systeme. Grundriß einer allgemeinen Theorie, Berlin/Frankfurt/M.: Suhrkamp 2003

Mau, Steffen: Das metrische Wir. Über die Quantifizierung des Sozialen, Berlin: Suhrkamp 2017.

Mauelshagen, Franz: Das Antlitz des Leviathan, in: Volkmer, Michael/Werner, Karin (Hg.): Die Corona-Gesellschaft. Analysen zur Lage und Perspektiven für die Zukunft, Bielefeld: Transkript 2020, 37-44. 
Nassehi, Armin: Muster. Theorie der digitalen Gesellschaft, München: C.H. Beck 2019.

Nassehi, Armin: Soziologe Nassehi: „Zerstreuung ist zivilisatorische Errungenschaft“ (Interview von Lisa Nimmervoll), in: Der Standard (26.03.2020), https:// www.derstandard.at/story/2000116158197/soziologe-nassehi-zerstreuung-ist-zivil isatorische-errungenschaft.

Offe, Claus: Krisen des Krisenmanagements. Elemente einer politischen Krisentheorie, in: Jänicke, Martin $(\mathrm{Hg})$ : Herrschaft und Krise. Beiträge zur politikwissenschaftlichen Krisenforschung, Opladen: Westdeutscher Verlag 1973, 197223.

Pfadenhauer, Michaela: Professionalität, Opladen: Leske+Budrich 2003.

Pfadenhauer, Michaela: Die Macht mediatisierter Kommunikation in persönlichen Öffentlichkeiten, in: Schröer, Norbert/Bidlo, Oliver/Keysers, Verena/Roslon, Michael (Hg.): Kommunikationsmacht, Weinheim: Beltz Juventa 2021a, 59-68.

Pfadenhauer, Michaela: Von entrückter Gewissheit zum gewissen Etwas. Eine Hinleitung zur Wissenssoziologie, in: Dimbath, Oliver/Pfadenhauer, Michaela (Hg.): Gewissheit. Beiträge und Debatten zum 3. Sektionskongress der Wissenssoziologie, Weinheim: Beltz Juventa 2021b, 20-29.

Poferl, Angelika: Wissensrelationen. Einführung in das Kongressthema, in: Poferl, Angelika/Pfadenhauer, Michaela (Hg.): Wissensrelationen. Beiträge und Debatten zum 2. Sektionskongress Wissenssoziologie, Weinheim: Beltz Juventa 2018, 23-28.

Scheller, Benjamin: Die Pest, die spanische Grippe und eine seltsame Niederlage. Vom Nutzen und Nachteil historischer Analogien in Zeiten von Covid-19, in: Florack, Martin/Korte, Karl-Rudolf/Schwanholz, Julia (Hg.): Coronakratie. Demokratisches Regieren in Ausnahmezeiten, Frankfurt/New York: Campus 2021, 257-268.

Schütz, Alfred/Luckmann, Thomas: Strukturen der Lebenswelt, Frankfurt/M.: Suhrkamp 1979.

Steg, Joris: Was heißt eigentlich Krise?, in: Soziologie 49/4 (2020) 423-435.

Turner, Victor: The Ritual Process. Structure and Anti-Structure, New Brunswick/ London: Aldine Transaction 2008.

Vobruba, Georg: Einfachdenken in der komplexen Gesellschaft. Das Volk, die repräsentative Demokratie und der Populismus, in: Endreß, Martin/Nissen, Silke/Vobruba, Georg: Aktualität der Demokratie. Strukturprobleme und Perspektiven, Weinheim: Beltz Juventa 2020, 105-155.

Weyer, Johannes: Krisenmanagement. Steuerungsfähigkeit des Staates und Akzeptanz der Bevölkerung, in: Soziologische Perspektiven auf die Corona-Krise. Digitales Kolloquium am 21.10.2020, Wissenschaftszentrum Berlin für Sozialforschung, https://coronasoziologie.blog.wzb.eu/podcast/johannes-weyer-krisen management-steuerungsfaehigkeit-des-staates-und-akzeptanz-der-bevoelkerung/. 
